\title{
Attachment of Tumor Cells to the Micropatterns of Glutaraldehyde (GA)-Crosslinked Gelatin
}

\author{
Yu-Cheng Ou, Chih-Wen Hsu, Lung-Jieh Yang ${ }^{1, *}$, \\ Hsieh-Cheng Han², Yi-Wen Liu² and Chien-Yuan Chen² \\ Department of Mechanical \& Electro-Mechanical Engineering, Tamkang University, \\ \#151, Ying-Chan Rd., Tamsui, 25137, Taiwan, Republic of China \\ ${ }^{1}$ National Applied Research Laboratories, \\ 3F, \#106, Ho-Ping E. Road, Sec. 2, Taipei, 10622, Taiwan, Republic of China \\ 2Institute of Microbiology and Biochemistry, National Taiwan University, \\ \#1, Sec. 4, Roosevelt Rd., Taipei, 10617, Taiwan, Republic of China
}

(Received July 30, 2008; accepted November 23, 2008)

Key words: gelatin, glutaraldehyde, tumor cells, attachment

In this work, we propose a novel technique for inducing the attachment of tumor cells to the micropatterns of glutaraldehyde (GA)-crosslinked gelatin. It provides another method to crosslink gelatin other than using photosensitizing agents or selective GA crosslink techniques. This novel technique can ensure the degree of crosslink, prevent an over-crosslink from pattern deformation and enhance the adhesion between gelatin and a glass slide. The best spatial resolution of micro-gelatin bases can be 2 $\mu \mathrm{m}$. The micropatterns of GA-crosslinked gelatin can still be formed successfully by conventional photolithography. The much less toxic and more biocompatible approaches of strengthening gelatin microstructures can be developed according to the idea herein.

\section{Introduction}

\subsection{Research motivation}

The development of tools for the manipulation of single cells in a parallel way is a very attractive issue for the rapid growing areas of genomics and proteomics. Thin film electrode arrays have been demonstrated in order to monitor the electrical signals generated from cells in vitro.(1) However, the adhesion of the monitored cells on the desired electrode is randomly distributed, and difficulties are encountered in monitoring many cells in parallel. A microrobot based on a polypyrrole-gold bilayer actuation has provided a potential tool for handling a single cell by moving arms gently and precisely.(2) The integration complexity of the microrobot system is high, but the minimum size of cells that the microrobot can grasp depends on the spatial resolution of the biocompatible microfabrication technology.

*Corresponding author: e-mail: Ljyang@mail.tku.edu.tw 
In recent years, gelatin has been a popular material and broadly introduced into cell and tissue engineering owing to its biocompatibility and easy gelation by changing the temperature of its solution. ${ }^{(3)}$ Gelatin is a derivative of collagen, one of the most common extracellular matrix (ECM) proteins. The cell growth and its viability that drive morphogenesis in complex tissues are controlled through the modulation of cell binding to the extracellular matrix.(4) Based on the facts that hyaluronan is a ubiquitous component of the extracellular matrix of all connective tissues, the higher weight percentage of gelatin is good for cell growth in a hyaluronan-gelatin hydrogel film. ${ }^{(5)}$ The gelatin immobilized on poly(acrylic acid) can also significantly enhance cell adhesion. ${ }^{(6)}$ Meanwhile, the different shapes of protein can affect the extension and migration of tumor cells, ${ }^{(7)}$ and gelatin is a suitable biomaterial for drug release. ${ }^{(8)}$ In this work, we have attempted to explore the novel potential approach of using a substrate with gelatin micropatterns to attract tumor cells for the application of drug delivery in the future. The gelatin micropatterns fabricated firmly on the substrate have a small size, which is purposely comparable to cell size, and are supposed to produce a selective attraction effect during the stages of falling and attachment of cell culture. Another reason to use gelatin as the culture base of the cells is its surface property. It was empirically reported that a more hydrophilic substratum stimulates cell growth, ${ }^{(9)}$ and therefore, gelatin intrinsically fits the hydrophilic requirement.

\subsection{Gelatin}

Gelatin, a polymer made from natural sources, has been used for over 6,000 years. It is made of proteins (18 amino acids) and was previously regarded as a pure food. The low melting point of natural gelatin $\left(<50^{\circ} \mathrm{C}\right)$ allows it to be formed into any shape by gentle heating or cooking. The gelatin is a biodegradable, biocompatible material, first used as a protection layer for low-temperature surface micromachining in 2002.(10,11) Natural gelatin has the drawback of dissolving in an aqueous environment and therefore requires a crosslinking procedure using appropriate agents. After being added with a photosensitizer, e.g., potassium dichromate $\left(\mathrm{K}_{2} \mathrm{Cr}_{2} \mathrm{O}_{7}\right)$, the gelatin gel acts like a negatively toned photoresist and can be used to fabricate many micropatterns with good mechanical strength and good resistance to chemicals.

However, photosensitizers are always highly toxic, therefore forbidding practical applications in a biomedical environment, e.g., the cell culture incubator in this work. This pushed many biomaterial researchers to use other less toxic crosslinking agents, such as glutaraldehyde (GA), carbodiimide, dextran dialdehyde and genipin. Gelatin strengthened by these functional group agents has more superior characteristics, such as biocompatibility, mechanical strength, antiwater transmission and antiswelling. These characteristics are very suitable for the aqueous environment of the cell culture base. In 2005, selective GA-crosslinked gelatin patterns were successfully introduced into cell culture. ${ }^{(12)}$ The selective growing density of human mesenchymal stem cells on the gelatin patterns surpasses that on the glass substrate by $2-3$ orders of magnitude. 


\subsection{Comparison with other patterning techniques for proteins or collagens}

In 2004, a gelatin film was used as a mask for chitosan culture bases in a biolithographic technique.(13) Pattern transfer was accomplished by applying a heated stamp to shape the gelatin and selectively crosslink the underlying chitosan. The spatial resolution of biopatterns was limited to the mm range only. Compared with other guided microcell-culturing methods using polydimethylsiloxane (PDMS) substrates with contact-printed laminin, ${ }^{(14)}$ or using robotic protein printing on amino-silane-modified glass slides, ${ }^{(15)}$ or using a fibronectin self-assembled monolayer (SAM) on a patterned metal surface, ${ }^{(16)}$ the gelatin micropatterns in this work attached directly onto the glass slides without prior immobilization of a linker agent on a substrate.

Therefore, the technique has a more concise concept and is simpler to use in achieving a comparable protein pattern size smaller than $100 \mu \mathrm{m}$ for the cell culture. One reason for choosing gelatin as the working protein for cell culture is based on economic factors. The processing cost of the GA-crosslinked gelatin micropatterns depicted in this paper is much lower than those in other biological methods. Hence, the application potential of extending this technique to large-area, wafer-level processing is highly promising. Second, the chip size of gelatin micropatterns after substrate dicing can be adjusted to be as small as possible to reduce the number of cells required for seeding. Finally, the gelatin micropatterns, after proper crosslinking by GA solution, have the advantage of a longer shelf life than other patterned proteins before coming in contact with the cell culture.

\subsection{Comparison with other fabricated gelatin patterns}

Gelatin negative resist can be used to fabricate micropatterns directly and the line width can be precisely controlled. However, photosensitizers are always highly toxic, therefore forbidding practical applications in a biomedical environment, e.g., the cell culture incubator in this work. Moreover, the fabricated selective GA-crosslinked gelatin patterns were successfully introduced into cell culture. The toxicity of selective GA-crosslinked gelatin was tested by using mesenchymal stem cells. This technique provided another means to crosslink gelatin other than using photosensitizing agents. However, the lateral dimension error occurs in the crosslinking of the selective GAcrosslinked gelatin patterns. Therefore, the selective GA-crosslinking fabrication technique requires an appropriate control of time to reduce the over-crosslinking distance and to generate fine line width. By this reason, the use of gelatin patterns without good crosslinking or accurate size is difficult in the environment of cell culture for several days. For example, the selective GA-crosslinked gelatin patterns may detach from the substrate, and the best spatial resolution of the micro-gelatin bases can only be $10 \mu \mathrm{m}$.

In this work, a new fabrication technique for the GA-crosslinked gelatin patterns is developed. It provides another method to crosslink gelatin neither by using photosensitizing agents nor by a selective GA-crosslinked technique. This novel technique can ensure the desired degree of crosslink, reduce the lateral dimension error, easily control the line width and enhance the adhesion between the gelatin and the glass slide. The GA-crosslinked gelatin patterns are compatible with the environment of cell culture. The best spatial resolution of the micro-gelatin bases can be $2 \mu \mathrm{m}$. 


\section{Materials and Methods}

\subsection{Fabrication of glutaraldehyde (GA)-crosslinked gelatin micropatterns}

In this paper, we have combined the concepts of GA crosslinking and conventional photolithography to fabricate gelatin microstructures with a biopatterning resolution as fine as $2 \mu \mathrm{m}$, and applied this technique to the cell culture. Such a small gelatin pattern cannot only be assigned as the biocompatible platform for cell culture but also has a size comparable to that of cells so as to possibly attract a very confined number of cells or even a single cell on the gelatin micropatterns both individually and in parallel.

The fabrication process for GA-crosslinked gelatin micropatterns is depicted in Fig. 1. First, we spin-coated a gelatin film on a glass substrate. (Preparation of pure gelatin: Dissolve gelatin powder in water at $40-50^{\circ} \mathrm{C}$. Filter to remove bubbles. Apply to glass plates by spin coating at $50^{\circ} \mathrm{C}$. Dry at room temperature for 3-4 h.) Second, we dipped the sample in $50 \%$ GA solution to conduct whole-wafer crosslinking with sufficient reaction time. Third, a masking layer of positively toned photoresist (e.g., AZP-4620) was spun on the gelatin surface, and the correlated ultraviolet (UV) exposure (365 nm, $16.5 \mathrm{~mW}$, for a minimum of $15 \mathrm{~s}$ ) of a portion of the photoresist defines the crosslinked gelatin etched with the $\mathrm{O}_{2}$ plasma. Finally, the glass substrate with a positively toned

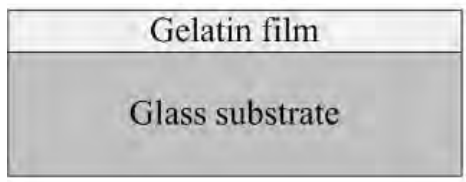

(a)

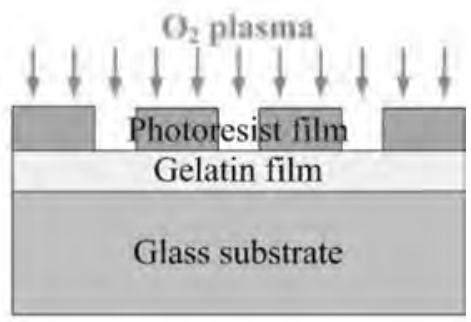

(c)

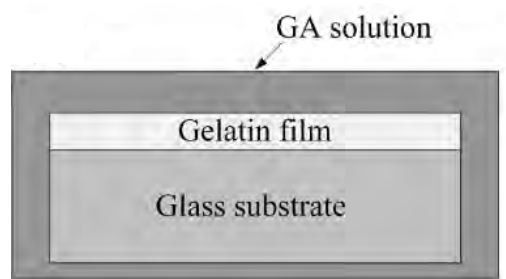

(b)

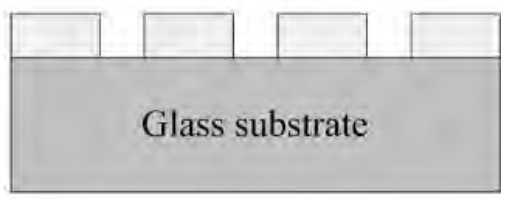

(d)

Fig. 1. Patterning process for GA-crosslinked gelatin: (a) spin coating of pure gelatin film at $50^{\circ} \mathrm{C}$ and drying for $1 \mathrm{~d}$, (b) crosslinking of gelatin film in $50 \%$ GA solution, (c) patterning of positively toned photoresist mask on the gelatin film and etching with $\mathrm{O}_{2}$ plasma, and (d) stripping of photoresist with acetone. 
photoresist mask on the GA-crosslinked gelatin film was etched with the $\mathrm{O}_{2}$ plasma (recipe: the etching rate of the GA-crosslinked film is shown in Table 1 and the etching rate of the positive photoresist, AZP-4620, is shown in Table 2). A reactive ion etching system (Model RIE-1C, SAMCO International Inc.) is employed here. The flow rate is $30 \mathrm{sccm}$ and the power is $80 \mathrm{~W}$. The average etching rate of the GA-crosslinked film is $0.2664 \mu \mathrm{m} / \mathrm{min}$ and that of the positive photoresist, AZP-4620, is $1.0068 \mu \mathrm{m} / \mathrm{min}$. In this $\mathrm{O}_{2}$ plasma etching process, the thickness of the GA-crosslinked film is controlled to $0.8 \mu \mathrm{m}$ and the thickness of the positive photoresist is controlled to $4 \mu \mathrm{m}$. Although the average etching rate of the positive photoresist is higher than that of the GA-crosslinked film, the GA-crosslinked micropatterns can still be protected from $\mathrm{O}_{2}$ plasma etching by thickness control. Finally, the GA-crosslinked micropatterns are obtained after stripping the masking layer of photoresist with acetone.

The fabricated gelatin patterns are shown in Fig. 2. Figure 2(a) shows the photoresist mask on a gelatin micropattern. Figure 2(b) shows the photoresist mask stripped with acetone and the GA-crosslinked gelatin micropatterns. It was found that no crack is produced on the GA-crosslinked gelatin micropatterns. It was proved that the $\mathrm{O}_{2}$ plasma process has no effect on the gelatin patterns. To measure the finest line width and the corresponding thickness of the fabricated gelatin micropattern, atomic force microscopy (AFM) in the noncontact mode is introduced to scan the surface profile of the gelatin micropattern shown in Fig. 3.

Table 1

Etching rate of the GA-crosslinked film ( $\mu \mathrm{m} / \mathrm{min})$.

\begin{tabular}{cc}
\hline Item & Etching rate \\
\hline GA-crosslinked film 1 & 0.2908 \\
GA-crosslinked film 2 & 0.2869 \\
GA-crosslinked film 3 & 0.2474 \\
GA-crosslinked film 4 & 0.2600 \\
GA-crosslinked film 5 & 0.2664 \\
GA-crosslinked film 6 & 0.2469 \\
\hline
\end{tabular}

Table 2

Etching rates of the positive photoresist samples, AZP-4620 $(\mu \mathrm{m} / \mathrm{min})$.

\begin{tabular}{cc}
\hline Item & Etching rate \\
\hline Sample 1 & 0.9935 \\
Sample 2 & 1.0000 \\
Sample 3 & 1.0005 \\
Sample 4 & 1.0075 \\
Sample 5 & 0.9910 \\
Sample 6 & 1.0480 \\
\hline
\end{tabular}



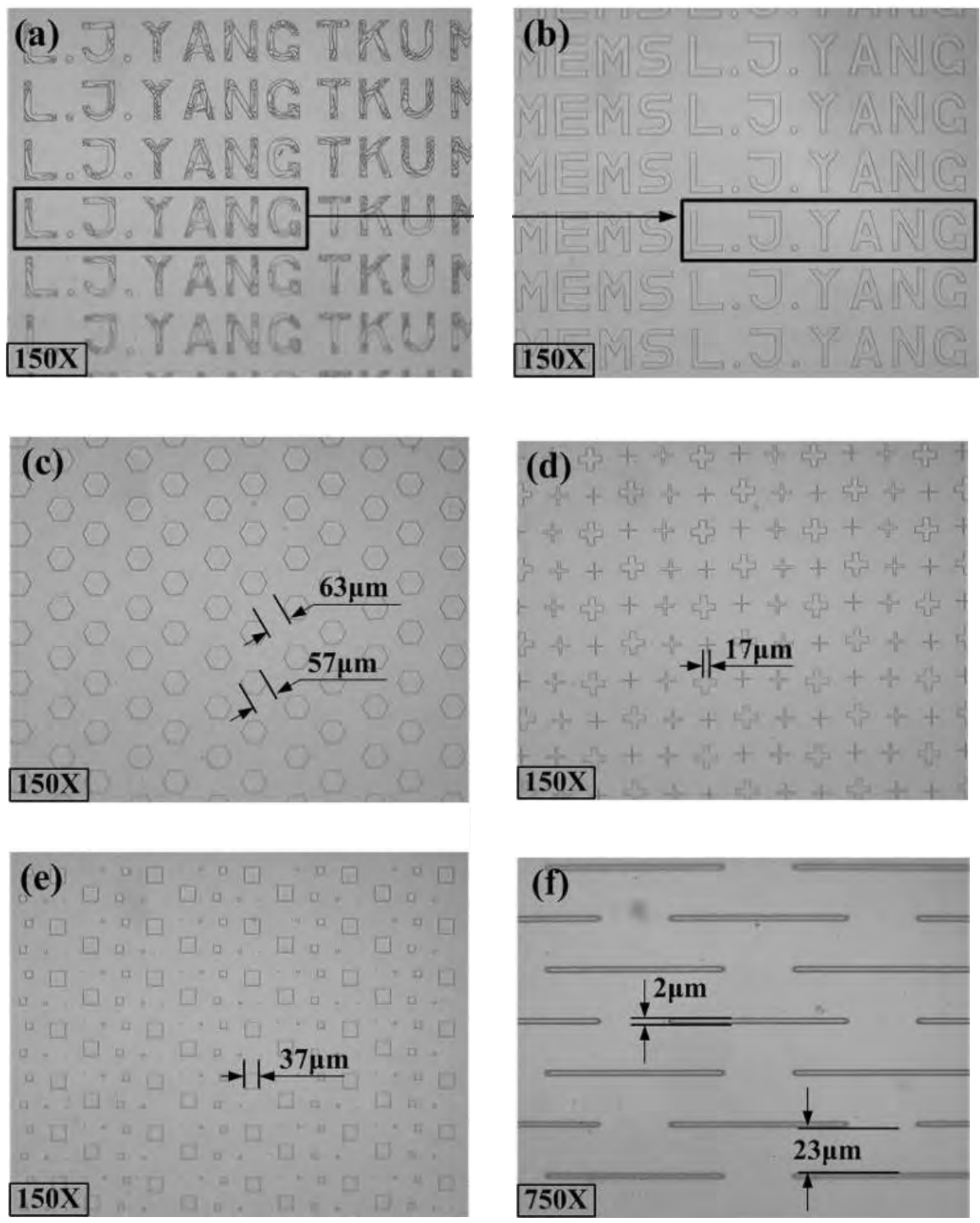

Fig. 2. Fabricated gelatin micropatterns: (a) before photoresist mask stripping, (b) after photoresist mask stripping, micro-letter pattern of $17 \mu \mathrm{m}$ width, (c) micro-honeycomb patterns, (d) micro-"cross" patterns with the largest width of $17 \mu \mathrm{m}$, (e) micro-square patterns with the largest width of $37 \mu \mathrm{m}$, (f) microstrip lines with width of $2 \mu \mathrm{m}$, length of $90 \mu \mathrm{m}$ and line gap of $23 \mu \mathrm{m}$. 

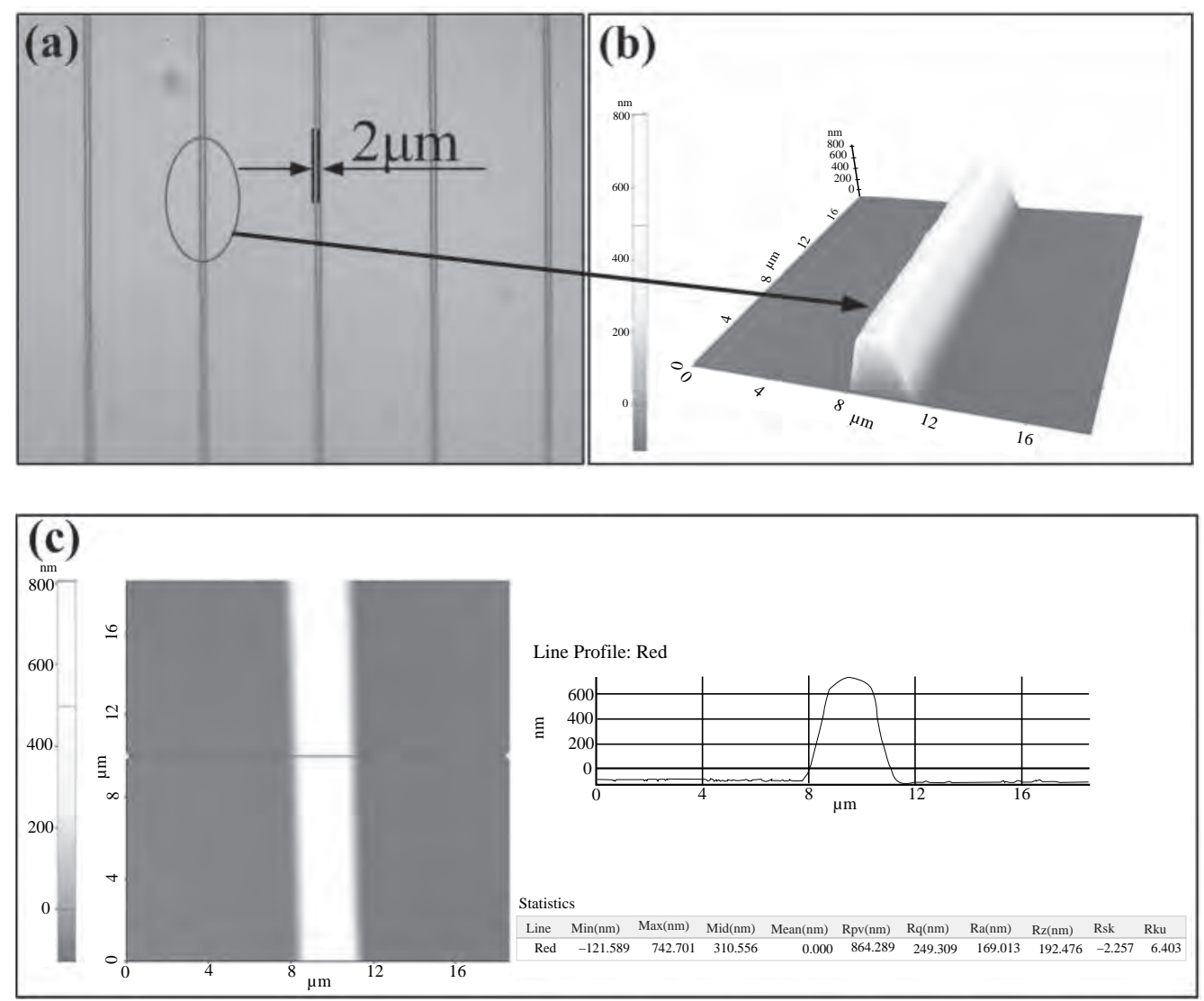

Fig. 3. Thickness measurement of gelatin micropattern scanned by AFM: (a) the scanned sample of microstrip lines of $2 \mu \mathrm{m}$ width, (b) AFM scanning image of microstrip gelatin, and (c) the crosssectional line profile of microstrip lines of $0.8 \mu \mathrm{m}$ thickness.

\subsection{Contact angle of glutaraldehyde (GA)-crosslinked gelatin}

Another reason for using gelatin as the culture base of the cells is its surface property. It has been reported that a more hydrophilic substratum can stimulate cell growth and the hydrophilic surface of gelatin can significantly enhance the cell adhesion. In this work, the contact angle of the surface on a glutaraldehyde (GA)-crosslinked gelatin film is also measured. The measured contact angles of the GA-crosslinked gelatin film and glass substrate are shown in Fig. 4. The contact angle of the GA-crosslinked gelatin film is measured to be about $57^{\circ}$. The contact angle of the glass substrate is measured to be about $41^{\circ}$. The contact angles of the different samples are shown in Table 3 . The measurement shows that the average contact angle of the GA-crosslinked gelatin film is larger than that of the glass substrate. 

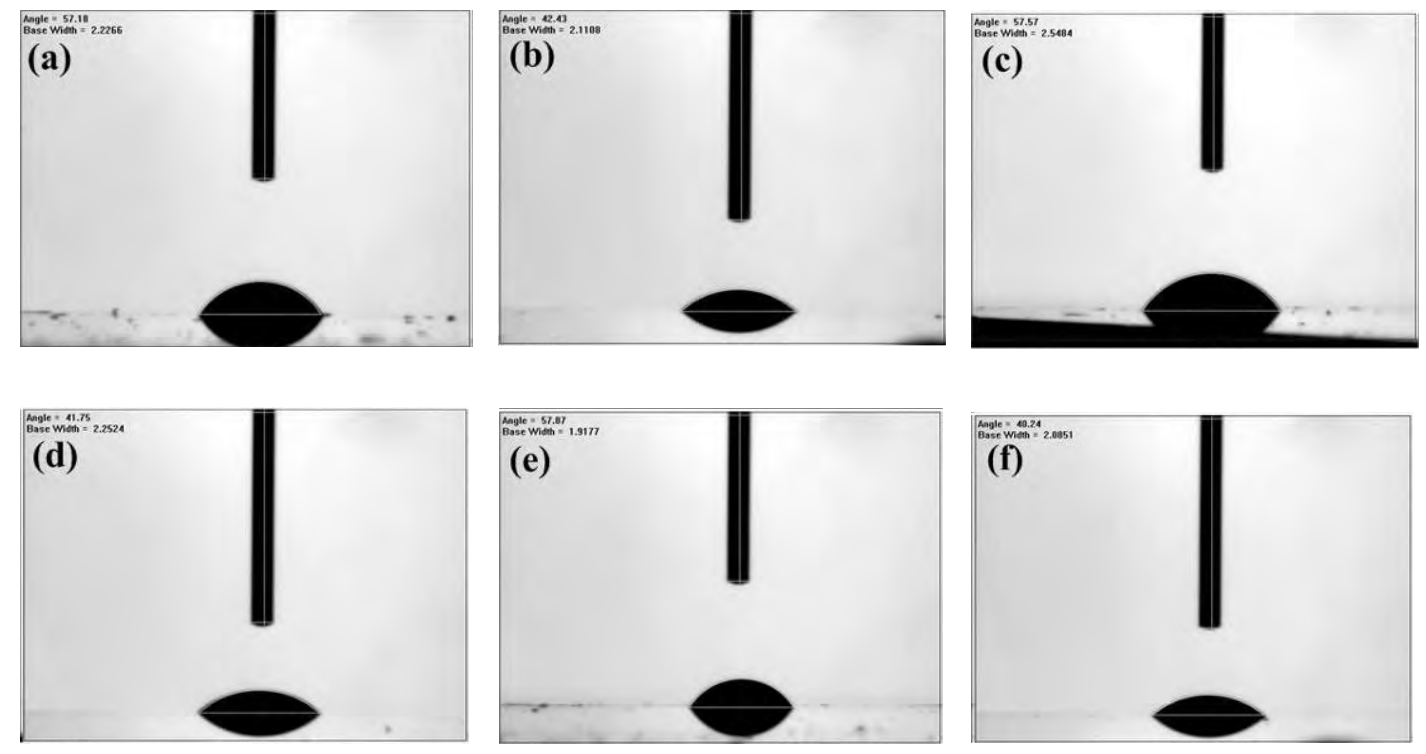

Fig. 4. Contact angle measurement: (a) GA-crosslinked gelatin film 1, (b) glass substrate 1, (c) GA-crosslinked gelatin film 2, (d) glass substrate 2, (e) GA-crosslinked gelatin film 3, and (f) glass substrate 3.

Table 3

Contact angles of the different samples.

\begin{tabular}{lc}
\hline \multicolumn{1}{c}{ Item } & Contact angle \\
\hline GA-crosslinked gelatin film 1 & $57.18^{\circ}$ \\
GA-crosslinked gelatin film 2 & $57.57^{\circ}$ \\
GA-crosslinked gelatin film 3 & $57.87^{\circ}$ \\
Glass substrate 1 & $42.43^{\circ}$ \\
Glass substrate 2 & $41.75^{\circ}$ \\
Glass substrate 3 & $40.24^{\circ}$ \\
\hline
\end{tabular}

\subsection{Swelling test for the aqueous environment of the cell culture}

Before using gelatin patterns as culture bases, the swelling effect of the medium to cell culture must be tested for practicability. Cell culture was then performed as follows. First, substrata with GA-crosslinked gelatin micropatterns were diced into slides. Second, the slides were subjected to UV sterilization and treated with phosphate buffered saline (PBS). Third, the slides were cultured in a minimum essential medium (MEM) with $10 \%$ fetal bovine serum (FBS) at $37^{\circ} \mathrm{C}$ in $5 \% \mathrm{CO}_{2}$ humidified atmosphere. Finally, after 2-day, 3-day and 6-day culture, the slides with GA-crosslinked gelatin micropatterns were observed by phase-contrast microscopy. The results of sequential soaking in the medium are shown in Fig. 5. pH is also measured to be about 7.64. There is no obvious 


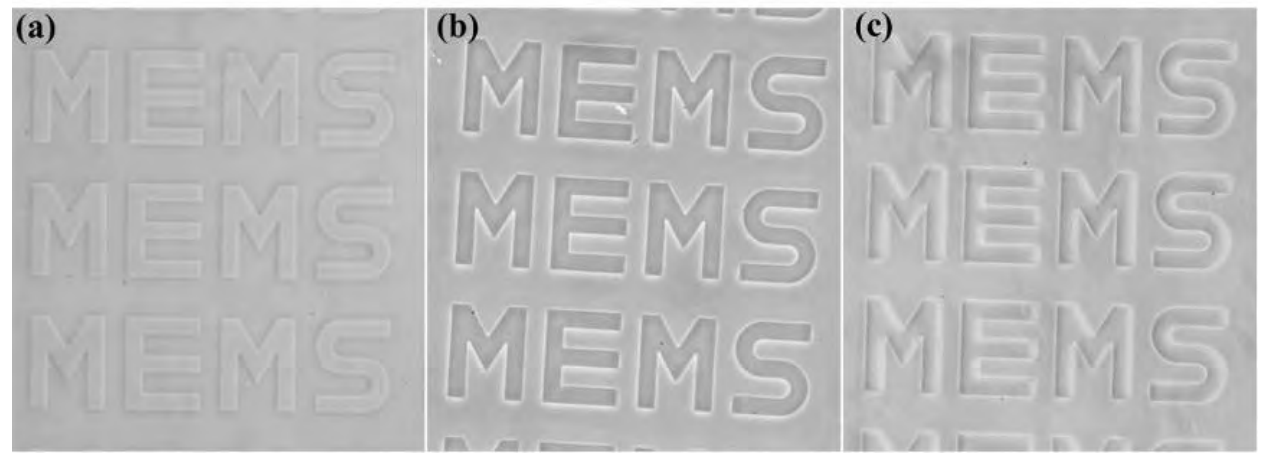

Fig. 5. Swelling effect of sequential soaking in the medium: (a) soaking for $2 \mathrm{~d}$, (b) soaking for $3 \mathrm{~d}$, and (c) soaking for $6 \mathrm{~d}$.

swell in the GA-crosslinked gelatin micropatterns. This ensures the practicability of the GA-crosslinked gelatin micropatterns, indicating that the micropatterns can be successfully introduced into the culture base.

\section{Results and Discussion}

In $\mathrm{O}_{2}$ plasma etching, it was found that the lateral dimension of all GA-crosslinked gelatin micropatterns decreases to about $3 \mu \mathrm{m}$ compared with the original design of the photomask. To obtain the correct line width and reduce the lateral dimension error, the line width of the photomask is modified according to the lateral dimension error caused by $\mathrm{O}_{2}$ plasma etching.

The different GA-crosslinked gelatin micropatterns are fabricated to observe cell extension. The behavior of the tumor cells growing on the gelatin micropaterns preliminarily observed by phase-contrast microscopy varies and depends on the type and properties of the cells, e.g., cell attachment, migration, proliferation, and morphology. In this work, the NS-1, HepG2 and HeLa cells are first used in the observation of attachment induced by GA-crosslinked gelatin micropatterns.

The HepG2 cells attached to the gelatin micropatterns are shown in Fig. 6. The HeLa cells growing on the gelatin micropatterns are shown in Fig. 7, indicating the growth of tumor cells on the side wall of the gelatin micropatterns during the falling and attachment of cell culture. Furthermore, the growth of tumor cells can extend in accordance with the shape of the micropatterns along the side wall. Due to the scale of the micropatterns, it was also found that two sides of tumor cells anchor the side walls between the different gelatin micropatterns. The NS-1 cells attached to the gelatin micropatterns are shown in Fig. 8. The NS-1 cells attach to the side walls and then directly divide. No obvious cell migration occurs in the NS-1 cells. 

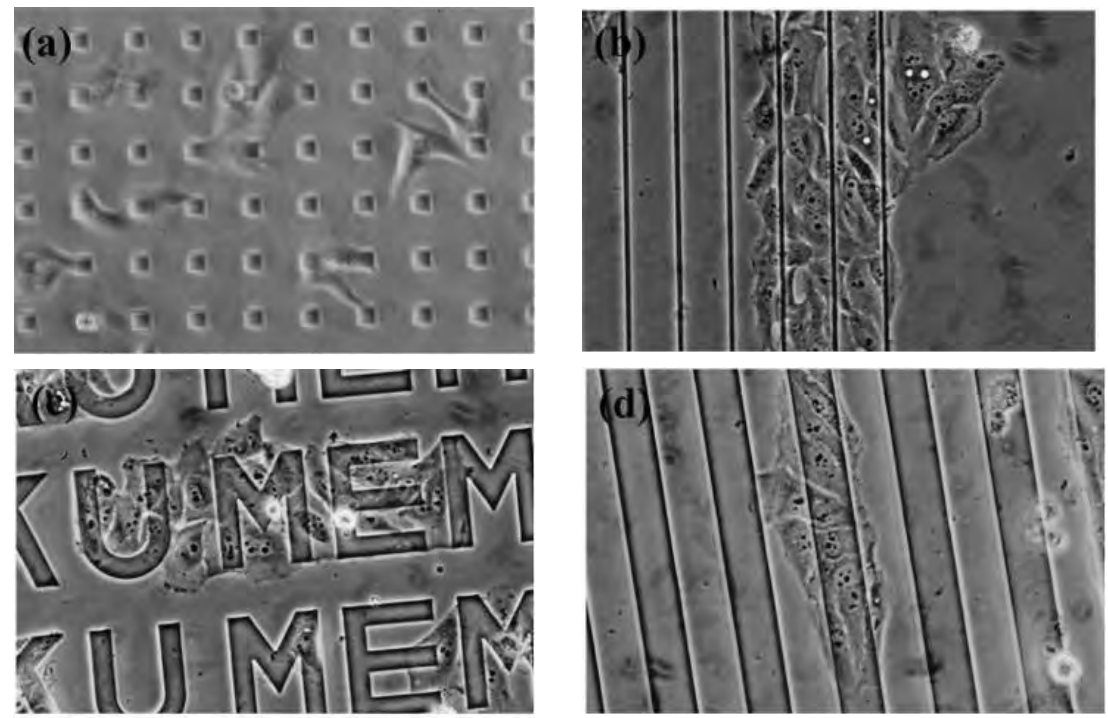

Fig. 6. Attachment of the HePG2 cells to the gelatin micropatterns: (a) the HePG2 cells attach to the micro-square patterns, (b) the HePG2 cells attach to the gelatin lines of $2 \mu \mathrm{m}$ width and grow along the gelatin lines, (c) the HePG2 cells attach to the different micro-"word" patterns, and (d) the HePG2 cells attach to the gelatin lines of $37 \mu \mathrm{m}$ width.
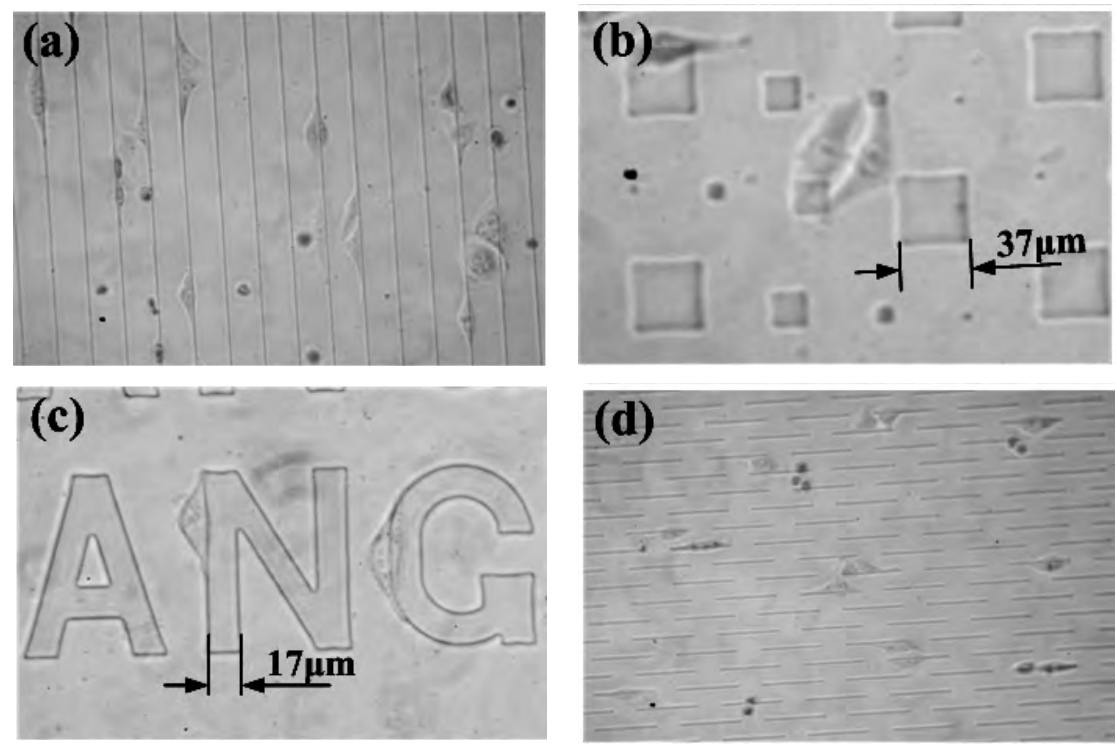

Fig. 7. Attachment of the HeLa cells to the gelatin micropatterns: (a) the HeLa cells attach to the gelatin with the tendency of extending to the shape of microstrip, (b) the HeLa cells anchor the side walls between the different gelatin micropatterns, (c) single HeLa cells attach to side walls of the micro-letter patterns, and (d) the HeLa cells attach to the gelatin and extend to the lines of 2 $\mu \mathrm{m}$ width and $90 \mu \mathrm{m}$ length. 


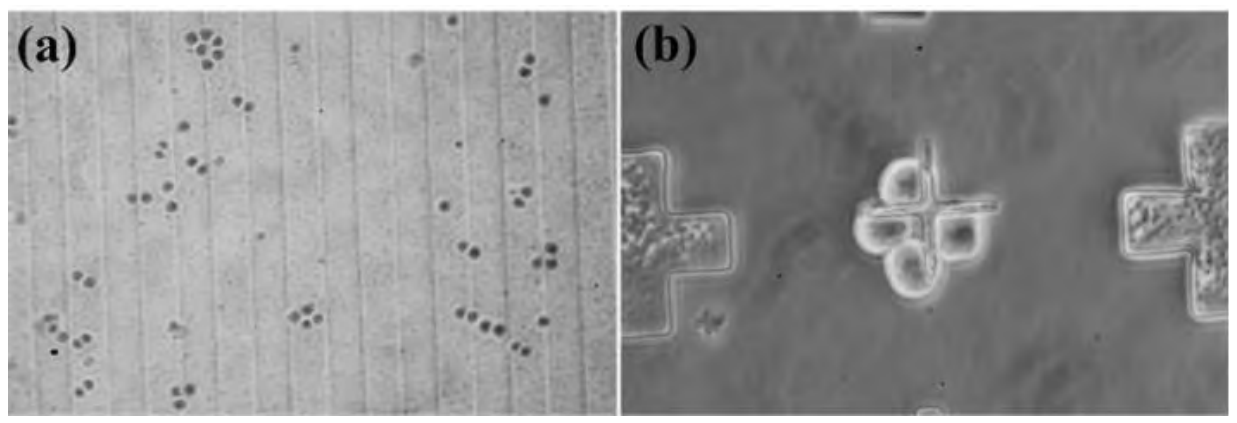

Fig. 8. Attachment of the NS-1 cells to the gelatin micropatterns: (a) the NS-1 cells attach to the microstrip lines, and (b) the NS-1 cells attach to the "cross” pattern.

\section{Conclusions}

The patterning and fabricated sample chips of GA-crosslinked gelatin are studied and modified in this paper. The micropatterns of GA-crosslinked gelatin can be formed successfully by conventional photolithography. The best spatial resolution of microgelatin bases can be achieved and adjusted to a size of $2 \mu \mathrm{m}$. Different GA-crosslinked gelatin micropatterns are fabricated to observe cell adhesion, extension and migration. The hydrophilic surface property of GA-crosslinked gelatin is preliminarily measured and confirmed on the basis of the contact angle of the GA-crosslinked gelatin film, which is $57^{\circ}$. The swelling effect of the medium to cell culture is also tested and no obvious swelling is found in the GA-crosslinked gelatin micropatterns. The practicability of using the GA-crosslinked gelatin micropatterns as a culture base is established. The behavior of the tumor cells varies and depends on the type and properties of the cells, e.g., cell attachment, migration, proliferation, and cell morphology. In the future, we believe that this biocompatibility, mechanical strength, chemical resistance, antiwater transmission and antiswelling of the cross-linked gelatin micropatterns will provide useful microstructural techniques for tumor cells, e.g., controlling tumor cells onto biosensors and exploring the effects of drug delivery to tumor cells.

\section{Acknowledgment}

The authors would like to thank the financial support of the Academic Technology Development Program of Republic of China (Taiwan ROC) with the research project numbers 94-EC-17-A-05-S1-017 and the National Science Council of Republic of China (Taiwan ROC) with the research project numbers NSC-96-2221-E-032-013, -014, -52-MY2. 


\section{References}

1 A. Mohr, W. Finger, K. J. Foehr, W. Goepel, H. Haemmerle and W. Nisch: Sens. Actuators, B 34 (1996) 265.

2 E. W. H. Jager, O. Inganaes and I. Lundstroem: Science 288 (2000) 2235.

3 S. M. Tosh and A. G. Marangoni: Appl. Phys. Lett. 84 (2004) 4242.

4 C. S. Chen, M. Mrksich, S. Huang, G. M. Whitesides and D. E. Ingber: Science 276 (1997) 1425.

5 X. Z. Shu, Y. Liu, F. Palumbo and G. D. Prestwich: Biomaterials 24 (2003) 3825.

6 H. Liu and Y. Ito: Lab Chip 2 (2002) 175.

7 M. Thery, A. Pepin, Y. Chen and M. Bornens: Proc. $\mu$ TAS'05 (TRF, Boston, 2005) p. 545.

8 W. F. Lee and S. C. Lee: J. Mater. Sci.: Mater. Med. 18 (2007) 1089.

9 M. S. Godbole, A. Seyda, J. Kohn and T. L. Arinzeh: Proc. IEEE 30th Annual Bioengineering Conference (IEEE, Northeast, 2004) p. 116.

10 L. J. Yang, W. Z. Lin, T. J. Yao and Y. C. Tai: Proc. 15th IEEE MEMS Conference (IEEE, Las Vegas, 2002) p. 471.

11 L. J. Yang, W. Z. Lin, T. J. Yao and Y. C. Tai: Sens. Actuators, A 103 (2003) 284.

12 L. J. Yang and Y. C. Ou: Lab Chip 5 (2005) 979.

13 R. Fernandes, H. Yi, L. Q. Wu, G. W. Rubloff, R. Ghodssi, W. E. Bentley and G. F. Payne: Langmuir 20 (2004) 906.

14 M. N. De Silva, R. Desai and D. J. Odde: Biomed. Microdevices 6 (2004) 219.

15 A. Revzin, P. Rajagopalan, A. W. Tilles, F. Berthiaume, M. L. Yarmush and M. Toner: Langmuir 20 (2004) 2999.

16 M. Veiseh, B. T. Wickes, D. G. Castner and M. Zhang: Biomaterials 25 (2004) 3315. 